\title{
Experiencias en la Blogosfera. Reflexiones en el Uso del Blog Bitácora
}

\section{Susana Archondo Ormachea ${ }^{a}$}

\author{
Rebut: 23/11/2010 Acceptat: 03/12/2010
}

\section{Resumen}

La investigación presenta dos experiencias en las cuales se incorpora el blog como herramienta de comunicación, y además como una bitácora de enseñanza e investigación. Los blogs se diseñan, se crean, y se utilizan. Estos blogs surgen como respuesta a las necesidades institucionales, y como bitácora del investigador.

Las instituciones participantes han sido la Creu Roja de Tarragona ONG internacional, y Le Groupement d'Intérêt Public Education et Formation Tout au Long de la Vie ${ }^{1}$ (GIP EFTLV) dependiente del Ministerio de Educación de Francia.

La pregunta de investigación descriptiva pretendía observar "in situ" que sucedía en la relación formador/investigador/estudiante y tecnología. Para lo cual partimos del supuesto de que el formador/investigador debe enseñar las competencias de aprender a aprender, y competencias TIC. Pero para que puedan enseñar dichas competencias deben contar con ellas.

Con tal cometido se ha elegido el blog, como herramienta TIC, y como herramienta de investigación. Es decir, el blog como tecnología autoreflexiva, intracomunicacional, y de relacionamiento consigo mismo, y con los tutores de las prácticas.

Esta investigación se ha construido en la medida que va avanzando. Es por lo tanto "un proceso y un constructo" subjetivo en evolución y construcción. Al respecto Paul Watzlawick (1978:8) dice: «ll n'existe pas de réalité absolue, mais seulement des conceptions subjectives et souvent contradictoires de la réalité».

Surge del convencimiento en la relevancia que presentan las TIC en la educación al ser utilizadas como herramientas para almacenar, difundir, compartir, investigar, etc. unida a la preferencia temática personal y algunas experiencias laborales.

Palabras clave: Formador/Investigador. Herramientas TIC. Blog. Competencias TIC. E portafolio.

\footnotetext{
${ }^{a}$ Departament de Pedagogia - Universitat Rovira i Virgili
} 


\section{Abstract}

This investigation is constructed in the measure that is advancing. It is therefore " a process and a constructo " subjective in evolution and construction. In the matter Paul Watzlawick (1978:8) says: "Il n'existe pas de réalité absolue, mais seulement des conceptions subjectives et souvent contradictoires de la réalité ».

It arises from the conviction in the relevancy that the TIC present in the education on having been used as tools to store, to spread, to share, to investigate, etc. Joined the thematic personal preference and some labor experiences.

The investigation presents two experiences in which the blog joins as tool of communication, and in addition as a binnacle of education and investigation. The blogs are designed, are created, and are in use. These blogs arise as response to the institutional needs, and as binnacle of the investigator.

The institutions participants have been the "Creu Roja" of Tarragona international NGO, and the "Groupement d'Intérêt Public Education et Formation Tout au Long de la Vie (GIP EFTLV)" of the Department of Education of France.

The question of descriptive investigation was trying to observe "in situ" that it was happening in the relation formingly / investigative and technology. For which we depart from the supposition from that the forming / investigative one must teach the competitions of learning to learn, and competitions TIC. But in order that they could teach the above mentioned competitions they must possess them.

With such an assignment the blog has been chosen, as tool TIC, and as tool of investigation. That is to say, the blog as autoreflexive technology, intracomunicacional, and communicational with it himself, and with the tutors of the practices.

Key words: Trainer. investigative. Tools TIC. Blog. Competitions TIC. And portfolio.

\section{Iniciando el camino}

La importancia de las TIC en la actualidad es indiscutible. Tan sólo imaginar despertar un día sin electricidad, sin ordenadores, sin móviles, sin conexión a la red Internet, es como vivir una verdadera pesadilla comunicacional. Esto sucede porque las TIC hoy en día son parte de la vida, están presentes no sólo en el ámbito laboral, también lo están en la formación, y por supuesto en el ámbito social y cultural.

Me conecto y luego existo, la no conexión para quien está acostumbrado al uso de las TIC y a la red, es la incomunicación con el mundo, y como seres humanos biosicoespirituales tenemos la necesidad básica y natural de comunicarnos. Existimos en tanto y en cuanto nos comunicamos con el otro, porque "ser" es relacionarse, y las TIC potencian y prolongan nuestros sentidos. 
Carr (2008) señala como el "gran invento" de una época con toda la novedad que conlleva se convierte en algo funcional y habitual y modifica las relaciones sociales. Con el ejemplo de la bombilla de Edison, cuenta como dicho invento tecnológico cambió la forma y hábitos de vivir de las personas. A la llegada de la noche los miembros de una familia se reunían frente al fuego para contarse lo sucedido en el día. Con la bombilla estos momentos sociales dejan de ser, cada uno podía realizar sus propias actividades en su propio ambiente. Hoy en día con las TIC, podemos estar virtualmente junto a la familia, y físicamente lejos de ella. Conectados con el mundo, y con nuestras propias redes más allá del espacio físico que ocupemos

Además de la relevancia de las TIC de las que comentábamos en párrafos anteriores, la elección de ésta temática estuvo influenciada primero, en el rol de alumna y luego de formadora a distancia. Ese doble rol, en dos tiempos distintos nos ha permitido ser empáticos en el momento de entender el proceso de relacionamiento del formador y del alumno frente a la nueva tecnología.

Segundo, como actora integrante de una red, vivenciando la potencialidad del trabajo a partir de una comunidad. Cubriendo de alguna manera la soledad y el aislamiento del formador, del investigador y del alumno más allá de las distancias físicas.

Tercero, como ser social en comunidad actuando y participando de la red de amigos, de familia, de actividades culturales y lúdicas. En redes como Facebook, Twenti, Reserchgate, y otras que nos permiten relacionarnos a nivel familiar, profesional, como en el ámbito investigativo, etc.

Es decir, como formadores y dejando de ser tecnófobos o tecnófilos la importancia radica en conocer las TIC, usarlas pero sobre todo apropiarnos y empoderarnos de ellas. Lo que se puede lograr a través del conocimiento de la potencialidad del medio y de su uso e integración en la vida.

Por otro lado, se ha dejado de lado el tabú generacional, en el entendido de que son las nuevas generaciones las que parecieran poseer un chip especial para entender las tecnologías (nativos) a diferencia de las personas mayores o de otra generación. Las experiencias actuales demuestran que esa brecha generacional en el manejo de las TIC, está más en la mente de las personas y que puede ser superado.

La cultura se ha convertido en una cibercultura y el formador en ese contexto debe ser en términos de Bartolomé (2008) un "Formador Cibernauta" en un entorno de trabajo en equipo, basado en redes TIC de trabajo y sociales que potencian el conocimiento a través de una inteligencia colectiva.

Lo novedoso de la TICE es que permite una autoformación, pero también permite una formación grupal, a partir de la participación en red. Estamos frente al nacimiento de una nueva visión de mundo que deja de ser individualista, y se construye en red, donde conocimiento es un hecho más bien global, grupal y dinámico. 
Lugar donde el formador deja de ser "el profesor" y se convierte en un profesional del conocimiento, y donde su identidad se construye de una manera reflexiva y crítica, pero sobre todo se construye a partir de un grupo de pertenencia en constante mejoramiento y crecimiento, como debe ser la red que los incluya. Dejamos de ser esos seres aislados y nos construimos en tanto y en cuanto somos parte de la red; existimos de manera individual pero como en la pirámide de Maslow requerimos de ese reconocimiento social que nos reafirma como seres individuales y como seres grupales. Porque en definitiva el ser humano sigue siendo esa unidad biosicoespiritual que existe en tanto que se relaciona y se construye con el "otro".

El nuevo rol del formador se encuentra mucho más claro, ya no es el clásico docente de atrio. El facilitador del proceso enseñanza/aprendizaje, no es quien todo lo sabe, es quien conoce los lugares donde encontrar conocimiento, y actualizarlo. Capaz de trabajar en red, tanto física como virtual, con sus pares a partir de aprendizajes significativos, multidisciplinares y con sentido. Lo que significa, enseñar a pensar, a razonar, a sistematizar, a poseer un sentido crítico, enseñar a trabajar en red, a ser creativos, enseñar en valores y ética. Que en otras palabras es un formador con la competencia de saber trabajar en red física y virtual, y tecnológicamente empoderado.

\section{Apuntes teóricos}

El fundamento teórico de la investigación se baso en tres ejes. El primer eje consta de las siguientes definiciones: Cultura Organizacional y Cibercultura. El segundo eje está formado por e Portafolio y Blogs. El tercer eje cuenta con teoría respecto al Formador cibernauta y Competencias TIC del formador.

Se entendera por cultura organizacional al "...sistema de significados compartidos por los miembros de una organización, que la distinguen de otras" (Robbins, 2004, p. 525).

Por otro lado Barbera (2008) considera la existencia de diversos tipos de e portafolio como ser: Académico (permite documentar eventos o productos relevantes para el autor de manera crítica y reflexiva, hace referencia a muestras de aprendizaje seleccionadas en el contexto escolar, universitario y formativo de manera general, puede ser: de profesores, de estudiantes). Profesional (documentar eventos o productos relevantes, de acuerdo al propósito y audiencia puede ser; profesional development, resume portfolio, showcase portfolio). Civil /empresarial. Vital (ámbito familiar, particular).

Barbera formula varias preguntas que perfilan el diseño de un e-portafolio como: ¿Para qué finalidad se desarrollará un e-portafolio y qué audiencia tendrá?, ¿Dónde se empleará el e-portafolio?, ¿Qué tipo de e-portafolio será y que ciclo vital tendrá?, ¿Se tienen en cuenta los diferentes medios de presentación (audio, vídeo, gráficos...) y los distintos tipos de información?, ¿Qué elementos-competencias, estándares, criterios...-- estarán implicados?, ¿Qué competencias tecnológicas específicas se 
necesitarán para manejarlo?, ¿Qué nivel de argumentación y reflexión se aplicará y cómo?, ¿Qué rol desarrollará la interactividad tecnológica y pedagógica?, ¿Cuándo se podrá consultar el e-portafolio y quién podrá hacerlo?, ¿Cómo se organizará la información? (p.38).

Los portafolios les permiten a los docentes registrar, evaluar y mejorar su trabajo. Son colecciones de trabajo especializados y orientados hacia un objetivo, que captan un proceso imposible de apreciar plenamente a menos que uno pudiera estar dentro y fuera de la mente de otra persona. Los portafolios convalidan expectativas actuales y legitiman las metas futuras. Constituyen una historia en desarrollo. Son fluidos, aunque pueden congelar un momento y hacerlo ver como si tuviera un principio y un final definidos. Son los museos de nuestro trabajo y nuestro pensamiento, en los que se exhiben nuestros éxitos, experimentos y sueños. Los portafolios son espejos, aunque sean deformantes, de una realidad en evolución. Nos muestran lo que queremos ver y lo que querríamos no ver. Además de ser un reflejo del yo, los portafolios son un espejo profesional.

El éxito del blog, entendido como un registro personal en línea, puede deberse a múltiples factores inherentes a sus características y potencialidades. El uso de los blog permite que estos sean públicos o que tengan un carácter más restrictivo. Como un diario de campo compartido con los profesores/tutores.

En las experiencias que se presentan en esta investigación el blog no es utilizado como un elemento masivo, sino más bien reflexivo, y comunicativo con los profesores y/o tutores, y con los estudiantes.

\section{Formador cibernauta y Competencias TIC}

La irrupción de las TIC en la enseñanza dibuja la llegada de un nuevo paradigma educativo centrado en "el estudiante" como ser en construcción. En un escenario físico y virtual, donde el aula universitaria trasciende y se convierte en un mix, un verdadero "blended learning", con mayor o menor grado de virtualidad. Escenario donde el facilitador se reconstruye y comienza a adquirir nuevas competencias. Porque, en esta sociedad del conocimiento en construcción, "el facilitador" es también un estudiante, y un ser en formación permanente.

Las habilidades, los roles, y las competencias TIC del formador han sido estudiadas y delimitadas por distintos investigadores, entre ellos podemos citar a Castell, Marqués, Bartolomé, Le Boterf, etc. En España una investigación reciente respecto a las competencias TIC para la docencia universitaria dirigida por Ma. Paz Prendes (2009) ${ }^{2}$ permite ver de manera detallada una lista de competencias, y además nos permite evaluar nuestras competencias como docentes TIC, en la misma red.

Una manera resumida de ver y entender estas 39 competencias básicas, de acuerdo a Cano (2005) "...es observar las dimensiones que las agrupan" (p. 149). 
En todo caso vale la pena enfatizar que las competencias TIC poseen características instrumentales y transversales, pero también transformadoras. Transformadoras de Modelos comunicativos, de la construcción de uno mismo, y del proceso de enseñanza / aprendizaje.

\section{Dos instituciones, dos países, dos experiencias}

En los dos puntos anteriores se ha mencionado la importancia de las TIC en el ámbito de la formación, y se han citado algunos componentes teóricos. Ahora se explicará el desarrollo de dos prácticas institucionales realizadas bajo el paraguas del Master Mundusfor, Formación de Profesionales de la Formación URCA (Université de Reims Champagne Ardenne) y URV (Universitat Rovira i Virgili).

Al ser un master europeo, realizado en dos países, las prácticas que se requerían realizar tenían que ser también en dos instituciones de los países en cuestión. De esa manera, se han desarrollado las prácticas en La Creu Roja de Tarragona ${ }^{3}$ (CRT), y Le Groupement d'Intérêt Public Education et Formation Tout au Long de la Vie ${ }^{4}$ (GIP EFTLV).

Estas instituciones han sido observadas a partir de sus características específicas institucionales, como ser la cultura organizacional, la comunicación y las TIC que utilizan. Además se ha considerado que al realizar la práctica cada una de las instituciones encomendó al practicante una misión específica.

La práctica se inicio con la CRT en España y en la medida de su desarrollo se fue afinando y descubriendo componentes teórico/prácticos. A partir de la segunda práctica en GIP EFTLV en Reims Francia, se fortaleció y se delimitó aún más la experiencia.

Si bien nos referimos a dos instituciones diferentes, la primera una ONG internacional y la segunda un proyecto del Ministerio de Educación francés. Encontramos componentes en común que abarcan desde los datos sociodemográficos de los actores (edad de los consultores y formadores), hasta el empleo de las TIC, y una cultura organizacional democrática y participativa.

Cada una de las experiencias presentaron peculiaridades como: En la CRT donde se quería fortalecer el componente de relación e interacción entre formadores, lo cual se hizo a partir del diseño y la creación de dos blogs para dos ámbitos; Inmigración y Formación (primero en castellano y luego en catalán). En el GIP, se trabajó con una misión determinada y específica delimitada desde un principio, se creó un blog en castellano para mostrar la imagen y potencialidades del proyecto hacia el mercado hispanoamericano, y catalán.

En general la experiencia describió el uso de herramientas TIC en relación los actores participantes; practicante/TIC, formador/TIC, tutor/TIC, etc. Es decir que se ha realizado una aproximación a la relación de los actores y la tecnología. 
Si bien, desde un inicio de las prácticas se contó con un blog bitácora, a lo largo del desarrollo de las mismas se introdujeron otros blogs, que respondieron a la necesidad de encontrar un instrumento o herramienta de comunicación entre pares, y un blog para mostrar una imagen a un nuevo público.

La preocupación principal que guió la investigación fue la idea de que los formadores deben conocer, usar, apropiarse y empoderarse de las TIC, para luego transmitir ese saber. En todo caso es una primera aproximación al objeto de estudio.

\section{Objetivos de la Investigación}

\subsection{Objetivos Generales}

Describir el uso de los blogs como herramienta de comunicación intrapersonal, interpersonal, y de reflexión, del investigador (portafolio virtual).

Describir y comparar el uso del blog como herramienta de comunicación intrapares, que realizan actores de la formación en la CRT ámbitos de Formación y de Inmigración, y en el GIP EFTLV D2PC.

\subsection{Objetivos Específicos}

1. Analizar e implementar un blog bitácora, de carácter autoreflexivo (portafolio).

2. Analizar la relación entre el uso de las TIC y la cultura organizacional en relación al trabajo en red de los actores internos de la CRT y del GIP EFTLV D2PC.

3. Caracterizar el uso de una herramienta TIC (blog) en relación al trabajo en red de los actores internos de las dos instituciones analizadas (CRT y del GIP EFTLV D2PC).

\section{Diseño metodológico}

Las decisiones teóricas y metodológicas asumidas responden a un conjunto de circunstancias, perspectivas e influencias múltiples; desde la multiculturalidad, las experiencias de vida, el bagaje teórico-práctico de los tutores. Las necesidades de complementar, y corroborar los resultados obtenidos a partir de la triangulación de herramientas.

En ese sentido las herramientas e instrumentos utilizados han sido: Investigación bibliográfica, observación participante, y no participante, entrevistas, y encuestas. Estas dos últimas con carácter complementario y descriptivo. 


\begin{tabular}{|c|c|c|c|}
\hline MÉTODOS 5 & TÉCNICAS $^{6}$ & FUENTES & INSTRUMENTOS $^{7}$ \\
\hline $\begin{array}{l}\text { Los métodos particulares } \\
\text { empleados son: } \\
\text { - Descriptivo } \\
\text { - Comparativo }\end{array}$ & $\begin{array}{l}\text { - Cualitativo } \\
\text { 1. Investigación Bibliográfica } \\
\text { 2. Observación } \\
\text { 3. Observación Participante } \\
\text { 4. Entrevistas no presenciales } \\
\text { 5. Cuestionario }\end{array}$ & $\begin{array}{l}\text { Libros referentes al tema, } \\
\text { curso de Formación básica } \\
\text { institucional. } \\
\text { La CRT y su accionar } \\
\text { El GIP EFTLV y su accionar } \\
\text { Actores representativos } \\
\text { (fuentes primarias) } \\
\text { Formadores/voluntarios } \\
\text { Consultores / formadores } \\
\text { Voluntarios CRT }\end{array}$ & $\begin{array}{l}\text { Fichas bibliográficas } \\
\text { Cuadro de observación. Blog } \\
\text { (Diario o bitácora) } \\
\text { Guía de entrevista no } \\
\text { presencial Cuestionario }\end{array}$ \\
\hline
\end{tabular}

Cuadro No 1 - Metodología de la investigación

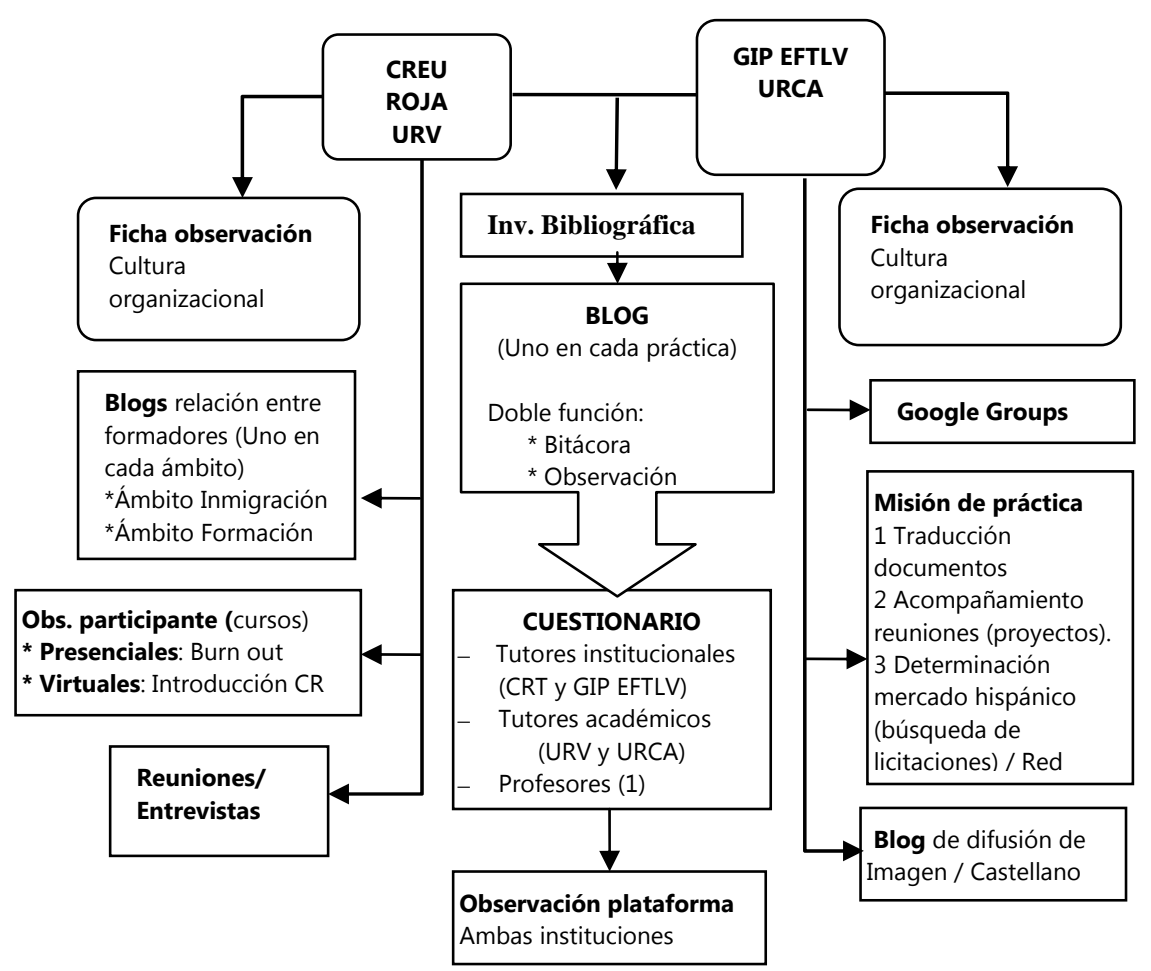

Cuadro № 2 - Diseño general de la investigación

A continuación se puede observar las fichas utilizadas para la observación no participante y participante. Por razones de espacio, colocaremos como ejemplo solamente la ficha de la CRT (Vale la pena señalar que para el GIP también se desarrollaron fichas). También se mencionará las entrevistas y encuestas realizadas de manera complementaria. 


\begin{tabular}{|l|l|}
\hline \multicolumn{2}{|l|}{ Etapas de observación no participante } \\
\hline \multirow{2}{*}{ Definición de Objetivos } & $\begin{array}{l}\text { Objetivo general: } \\
\text { Observar y determinar cuál es la cultura organizacional en el ámbito de } \\
\text { Formación y ocupación y Inmigración de la CRT. } \\
\text { Objetivos específicos: } \\
\text { Identificar si la orientación de la CRT se enfoca a: la innovación, la orientación a } \\
\text { los resultados, orientación a las personas, orientación a los equipos. } \\
\text { Determinar la comunicación empleada en la CRT, a partir de la comunicación } \\
\text { organizacional; formal, informal, interna, externa; en red o individual. }\end{array}$ \\
\hline Acceso al Escenario & $\begin{array}{l}\text { Se considera que es aconsejable el acceso en varios momentos de } \\
\text { observación.Seleccionar dimensiones de observación, tiempo, acontecimientos } \\
\text { de interés y lugares, escenario variable (ámbitos elegidas y determinadas, } \\
\text { intranet, Web). } \\
\text { Informales y formales. }\end{array}$ \\
\hline $\begin{array}{l}\text { Desarrollo } \\
\text { de la Observación } \\
\text { Salida del escenario }\end{array}$ & $\begin{array}{l}\text { La observación "in situ" se desarrolla empleando una ficha de observación y } \\
\text { registro, en varias sesiones aprovechando el desarrollo de la práctica. } \\
\text { Salir del escenario. }\end{array}$ \\
\hline
\end{tabular}

Cuadro $N^{\circ} 3$ - Cultura organizacional CRT

\section{Etapas de observación}

\begin{tabular}{|l|l|}
\hline \multirow{5}{*}{ Definición de Objetivos } & $\begin{array}{l}\text { Objetivo general: } \\
\text { Observar y determinar cuál es la cultura organizacional en el ámbito de } \\
\text { Formación y ocupación y Inmigración de la CRT. } \\
\text { Objetivos específicos: } \\
\text { Identificar si la orientación de la CRT se enfoca a: la innovación, la orientación a } \\
\text { los resultados, orientación a las personas, orientación a los equipos. } \\
\text { Determinar la comunicación empleada en la CRT, a partir de la comunicación } \\
\text { organizacional; formal, informal, interna, externa; en red o individual. }\end{array}$ \\
\hline Acceso al Escenario & $\begin{array}{l}\text { Se considera que es aconsejable el acceso en varios momentos de } \\
\text { observación. } \\
\text { Seleccionar dimensiones de observación, tiempo, acontecimientos de interés y } \\
\text { lugares, escenario variable (ámbitos elegidas y determinadas, intranet, Web). } \\
\text { Informales y formales. }\end{array}$ \\
\hline $\begin{array}{l}\text { Desarrollo } \\
\text { de la Observación } \\
\text { Salida del escenario }\end{array}$ & $\begin{array}{l}\text { La observación "in situ" se desarrolla empleando una ficha de observación y } \\
\text { registro, en varias sesiones aprovechando el desarrollo de la práctica. } \\
\text { Salir del escenario. }\end{array}$ \\
\hline
\end{tabular}

Cuadro Nº 4 - Observación Plataforma CRT 


\begin{tabular}{|l|l|}
\hline \multicolumn{2}{|c|}{ Etapas de observación participante } \\
\hline Definición de Objetivos & $\begin{array}{l}\text { Objetivo general: } \\
\text { Identificar y caracterizar el uso del "blog bitácora" por parte de los tutores } \\
\text { institucionales y académicos y de } 2 \text { docentes. } \\
\text { Objetivos específicos: } \\
\text { Observar la relación de los actores frente al blog. }\end{array}$ \\
\hline $\begin{array}{l}\text { Acceso al Escenario } \\
\text { de la Observación }\end{array}$ & $\begin{array}{l}\text { En varios momentos, consultas a los tutores y profesores y constatación en el } \\
\text { blog. }\end{array}$ \\
\hline \multirow{2}{*}{$\begin{array}{l}\text { La observación se desarrolla empleando una ficha de observación y registro, } \\
\text { donde el blog bitácora juega un papel muy importante. }\end{array}$} \\
\hline $\begin{array}{l}\text { Definición de Objetivos } \\
\text { Desarrollo la Observación }\end{array}$ & $\begin{array}{l}\text { Objetivo general: } \\
\text { Identificar y caracterizar el uso del "blog bitácora" por parte de los tutores } \\
\text { institucionales y académicos y de } 2 \text { docentes. } \\
\text { Objetivos específicos: } \\
\text { Observar la relación de los actores frente al blog. }\end{array}$ \\
\hline Acceso al Escenario & $\begin{array}{l}\text { En varios momentos, consultas a los tutores y profesores y constatación en el } \\
\text { blog. }\end{array}$ \\
\hline Lagabundeo & Labservación se desarrolla empleando una ficha de observación y registro, \\
\hline
\end{tabular}

Cuadro № 5 - Observación participación del "blog bitácora"

\begin{tabular}{|l|l|}
\hline \multicolumn{2}{|l|}{ Etapas de observación participante } \\
\hline \multirow{5}{*}{ Definición de Objetivos } & $\begin{array}{l}\text { Objetivo general: } \\
\text { Identificar las habilidades y estrategias concernientes a la facilitación o } \\
\text { intervención educativa. } \\
\text { Objetivos específicos: } \\
\text { Observar como interactúa el formador de la CRT. } \\
\text { Participar como alumna en un curso presencial de la CRT. } \\
\text { Comunicar objetivos, situación de partida verificada, comportamiento, } \\
\text { intervención, capacidad de diálogo, empatía, aporte de ideas, moderación, } \\
\text { autoconfianza, recursos, gestión del tiempo, métodos y técnicas, objetivos } \\
\text { finales evaluados. }\end{array}$ \\
\hline Acceso al Escenario & $\begin{array}{l}\text { En un momento dado, con un curso específico. } \\
\text { Seleccionar dimensiones de observación, tiempo, acontecimientos de }\end{array}$ \\
\hline Desarrollo & $\begin{array}{l}\text { La observación "in situ" se desarrolla empleando una ficha de observación y } \\
\text { registro. }\end{array}$ \\
\hline
\end{tabular}

Cuadro No 6 Curs de Les Estratègies de les tecniques de Burn Out: Posició del Tècnic 


\begin{tabular}{|l|l|}
\hline \multicolumn{2}{|c|}{ Etapas de observación participante } \\
\hline Definición de Objetivos & $\begin{array}{l}\text { Objetivo general: } \\
\text { Observar y participar como alumna virtual en un curso de la CRT } \\
\text { Objetivos específicos: } \\
\text { Caracterizar un recurso virtual empleado por la CRT. }\end{array}$ \\
\hline Acceso al Escenario & Aula virtual. \\
\hline $\begin{array}{l}\text { Desarrollo } \\
\text { de la Observación }\end{array}$ & $\begin{array}{l}\text { La observación "in situ" se desarrolla empleando una ficha de observación y } \\
\text { registro del curso on line. }\end{array}$ \\
\hline
\end{tabular}

Cuadro Nº 7 - Formación Básica Institucional

\section{Entrevistas y reuniones}

Para enriquecer los resultados se realizaron entrevistas no directivas en función de objetivos de conocimiento entre la institución y el investigador, a partir de una guía de preguntas, de carácter abierto y específico. Estas entrevistas han sido grabadas en su totalidad, sin embargo, no se ha realizado una transcripción textual, los aportes que se obtuvieron han sido revisados por los tutores institucionales y académicos.

Cada uno de los ítems o preguntas responde a objetivos específicos de conocimiento institucional y de servicio específicos. Por ejemplo en la entrevista a la tutora institucional y responsable del ámbito de Formación y Ocupación de la CRT se pretendió conocer los componentes de gestión educativa de calidad que se utilizan en ese ámbito, lo propio sucedió con la entrevista realizada con el responsable del ámbito de Inmigración.

Las entrevistas fueron validadas a partir del apoyo de los tutores. Luego se la aplicó a individuos elegidos previamente. En algunas oportunidades y con algunas personas se realizaron más de una entrevista, con el propósito de complementar y añadir datos.

Las personas entrevistadas fueron las siguientes: La Directora del Departamento de Capacitación, la Responsable del Ámbito de Formación y Ocupación, el Responsable del Ámbito de Inmigración y el Director Nacional de Educación a Distancia (Barcelona). Coordinadoras del programa de Ocupación (3 técnicos), para internalizarse en las funciones y actividades que realiza, lo que permitió complementar la información proporcionada a través de la documentación institucional proporcionada. Entrevistas con el Responsable de Formación oficina local, Técnico de Formación de Voluntarios, Técnico de espacio de investigación de trabajo, que permitió ver la actividad más de campo, más del día a día de la formación. Este último técnico permitió además revisar las especialidades virtuales y los recursos virtuales de la UOC. Con la administrativa de soporte se ingreso al intranet en el área de Formación y Ocupación.

Para iniciar la fase de entrevistas se ha realizado una exploración descriptiva y luego una fase intermedia con una guía de entrevistas o preguntas abiertas para recabar 
mayor información. Además, se han realizado cuestionarios a los tutores institucionales y académicos, y a dos profesores de la universidad para complementar los resultados obtenidos del uso del blog de bitácora.

\section{Encuestas}

Las encuestas planificadas se enfocaron a los voluntarios de la CRT con la intención de testear la opción de implementación de cursos virtuales y participación en el proceso de creación e implementación del blog (red y comunidad virtual).

El cuestionario ha sido contrastado por el jefe de voluntarios de la CRT y por la Responsable de Formación. Habiendo sido modificado de acuerdo a sus sugerencias, que principalmente señalaba el empleo de un lenguaje menos técnico y más directo. Se han obtenido respuesta de cuatro de nueve voluntarios.

\section{Desarrollo de la experiencia}

La planificación inicial de la experiencia comprendía 150 horas de práctica en cada institución. Es decir un total de 300 horas, las actividades y el desarrollo de la práctica sobrepasaron dicho horario. La dinámica generada permitió, en las dos organizaciones, una continuidad y un seguimiento virtual, hasta un semestre después de haber finalizado la actividad.

El blog utilizado como bitácora ha sido sistematizado y se evidencia a sí mismo. Se utilizó, como bitácora, un blog en cada práctica. "La investigación demostró que escribir diarios de aprendizaje refuerza, y desarrolla el alcance del aprendizaje del alumno en la práctica porque de acuerdo a Lea, M. Stieres (2000) "...en primer lugar, los diarios ofrecen la oportunidad de escribir de manera regular, lo que permite desarrollar las ideas y la fluidez en la escritura... En segundo lugar, un diario permite a los estudiantes construir un mapa de las relaciones y estructuras complejas de un curso o material variado... En tercer lugar, un diario hace que los alumnos piensen de forma distinta" (pp. 98-99).

Los blogs/bitácoras fueron creados al inicio de cada una de las prácticas para responder a tres objetivos; evidenciar el trabajo diario de la práctica, permitir el diálogo permanente con los tutores, iniciar los trabajos de fin de semestre y finales del master, y la redacción de artículos.

Los blogs que se utilizaron en un principio eran cerrados y restrictivos permitiendo solamente el seguimiento y participación de los tutores, y del estudiante de práctica. 
En un principio se pensó en la necesidad de la creación de dos blogs adicionales uno para el marco teórico, y el otro para la metodología de la investigación. Finalmente se decidió por la creación de un blog para el componente metodológico.

\section{7. "Hinc et nunc" Resultados y Conclusiones}

La frase Hinc et nunc significa aquí y ahora, y es como se quiere mostrar esta experiencia válida en el momento que se desarrolló.

Los objetivos tanto general, como específicos también se han cumplido. Es decir que se ha realizado un acercamiento descriptivo/comparativo del uso de las TIC, entendidas como herramientas de comunicación intrapares, que realizan actores de la formación en la CRT ámbitos de Formación y de Inmigración, y en el GIP EFTLV D2PC.

Además se ha investigado la relación entre el uso de las TIC y la cultura organizacional en relación al trabajo en red de los actores internos de la CRT y del GIP EFTLV D2PC.

En ambas prácticas se ha propuesto un instrumento para mejorar el proceso comunicacional entre pares, se ha construido los blogs, y se ha iniciado el proceso de dinamización del mismo.

La importancia de la comunicación y el uso de la red nos llevan a pensar en la necesidad de afianzar, y enfatizar la importancia que tiene la competencia de saber aprender a lo largo de la vida, de empoderase de las TIC y saber trabajar en red de manera física y tecnológica.

A continuación se muestran los resultados obtenidos, posteriormente a manera de interpretación se detallan las conclusiones a las que se ha arribado.

Se ha descrito el uso de blogs como herramienta comunicacional, a partir de la creación del blog/bitácora, que ha sido útil para la ordenación de ideas, seguimiento y evidenciación de actividades. Es decir que ha sido utilizado para la autorreflexión y automotivación. Seguimiento y control, y comunicación con los tutores (trabajo de investigación en red). Permitió agrupar artículos capaces de ser útiles en la redacción final del informe, pudiendo contar con el día a día de la práctica desarrollada. Es decir, que podemos indicar que la creación del blog/bitácora fortaleció el proceso comunicativo y evidenció su uso como herramienta de trabajo en red.

Las reuniones físicas con los tutores académicos e institucionales, el personal técnico de la CRT y del GIP EFTLV aportaron conocimientos, experiencia, profesionalidad y visión institucional. De esa manera se delimitó más el área de práctica. Reconfigurándose y adecuándose la metodología, los instrumentos, las herramientas y las necesidades de consultas teóricas y metodológicas. 
A partir de las prácticas desarrolladas en las dos instituciones hemos podido comparar, el mismo objeto; blog/bitácora, y la experiencia en el empleo del blog como herramienta de comunicación, y autoreflexión.

Veamos con más detalle cuáles han sido las diferencias y coincidencias encontradas. $\mathrm{Si}$ bien son dos instituciones con características diferentes, ambas trabajan en formación, y cuentan con formadores, y consultores, con un rango etáreo muy similar. Poseen una cultura organizacional abierta y fuertemente dirigida al trabajo grupal. La plataforma que utilizan es de última generación, y trabajan, o han empezado a trabajar con la formación a distancia (FOAD, E learning, blended learning, etc).

En ese sentido podemos decir que cuentan con recursos TIC para el relacionamiento "intrapares" como la posibilidad de crear o participar de foros, chats, correo electrónico, crear o participar de blogs, ingresar en grupos de trabajo (a partir de Google Groups), etc.

Cuentan con un problema común al introducir TIC, es que cuando los formadores están comprendiendo e introduciéndose en componentes TIC, se introducen cambios en las plataformas, que requieren de un constante aprender a aprender. Si bien son los expertos tecnológicos los que dominan está área y fortalecen los componentes, y ello es loable, sin embargo estamos frente a recursos humanos, o capitales humanos, que requieren de formación, motivación, seguimiento, y que además deben estar preparados ante la innovación y los cambios, etc.

Otro componente es la existencia consciente de un cambio de mentalidad del formador, que deja de ser ese "maestro tradicional" y se convierte en facilitador del proceso, dentro de la "sociedad red" que menciona Castells y conformando "comunidades virtuales", y que al igual que el proceso de formación se está reconfigurando a partir de las TIC. El formador debe adquirir competencias TIC y competencias red.

Y un tercer componente es que el formador siente que, en vez de que la tecnología es un elemento que le permitirá optimizar su tiempo, se convierte en un elemento que le quita tiempo, porque debe subir archivos, debe revisar los que se ha hecho virtualmente. La presencia de muchas plataformas, y muchos blogs, y la solicitud de participación activa de los formadores, genera una sensación de agobio informacional. Lo que significa, que se incrementa el trabajo. Es decir, que cada vez hay más claves que recordar, más links a los cuales se debe ingresar y se debe conocer, más información que procesar y se comienza a sentir los efectos de la "infoxicación". El formador se siente en un mundo lleno de información diversa y dispersa en la que navega y naufraga constantemente.

En los párrafos anteriores hemos hablado de componentes en común entre las dos instituciones de práctica. Ahora veamos las especificidades que se derivan directamente de la misión encomendada por cada una de instituciones. 
En el caso de la CRT, y luego de un diálogo con personal de la institución, y ha solicitud de ellos se han creado dos blogs para intensificar el relacionamiento inter voluntarios/formadores en dos secciones distintas, y se ha participado como alumna en la formación a distancia, y presencial, y luego se ha seguido el proceso como voluntaria.

En el caso del GIP EFTLV D2PC la misión contenía tres partes; traducción de documentos, acompañamiento a reuniones para conocer los procedimientos y finalmente, un análisis del mercado hispánico para la creación de una red. Que ha permitido desde el simple conocimiento de la institución, hasta el hecho de implicarse en las actividades y el posterior compromiso al contar con una misión específica de la cual ser era absolutamente responsable. Para el GIP EFTLV se ha creado un blog en castellano con el propósito de difundir la imagen de la institución y captar futuros socios. Por lo tanto las misiones encomendadas se han cumplido, y se prevé continuar en los próximos meses con el apoyo a la conformación de la red hispanoamericana.

\section{Las TIC aplicadas a las prácticas empresariales (blog/bitácora)}

Su creación ha sido muy útil para la ordenación de ideas, seguimiento y evidenciación de actividades, es decir que sirve para la autorreflexión y automotivación, seguimiento y control, y comunicación con los tutores (trabajo de investigación en red). Permite agrupar artículos capaces de ser útiles en la redacción final del informe. La creación del blog/bitácora fortaleció el proceso comunicativo y evidenció su uso como herramienta de trabajo en red.

Al finalizar la experiencia me pregunte, y pregunto al lector: ¿Cómo se puede ser conclusivo en un mundo de continuas transformaciones? ¿Dónde la constante es el cambio y las TIC evolucionan y transforman lo social, lo cultural, lo económico y lo político, y dónde la formación es un proceso a lo largo de la vida?

\footnotetext{
Notas

${ }^{1}$ En adelante GIP EFTLV.

${ }^{2}$ Estudio de Competencias TIC para la docencia en la Universidad Pública Española: Indicadores y propuestas para la definición de buenas prácticas que ha sido financiado por el Ministerio de Educación a través de la convocatoria para la subvención de acciones con cargo al programa de Estudio y Análisis. Universidad de Murcia.

${ }^{3}$ En adelante CRT.

${ }^{4}$ En adelante GIP EFTLV.

${ }^{5}$ El método es el camino que guía la investigación. Para Max Hermann en "Investigación económica su metodología y su técnica" p. 39, es la manera de alcanzar un objetivo; o bien, se le define como determinado procedimiento para ordenar la actividad. Existen métodos generales y
} 
particulares. Los segundos son los que cada disciplina ha desarrollado de acuerdo a sus necesidades y limitaciones.

${ }^{6}$ Se entiende como técnica al conjunto de reglas y operaciones para el manejo de los instrumentos que auxilia en la aplicación de los métodos.

${ }^{7}$ Permiten obtener datos sistemáticamente.

\section{Bibliografía}

Barberá, Elena. (2008). El estilo e-portafolio. Barcelona: Editorial UOC.

Bartolomé, Antonio. (2008). El Profesor Cibernauta ¿Nos ponemos las pilas? Barcelona: Editorial Graó de IRIF. S.L.

Carr, Nicholas. (2008). El gran interruptor. El mundo en red de Edison a Google. Ediciones Deusto.

Castells, Manuel. (2003). La era de la información. La societat xarxa. Barcelona: Editorial UOC.

Covey, Stephen. (2009). Los 7 hábitos de la gente altamente efectiva. Paidos Ibérica.

Le Boterf. (1999). L'ingenierie des compétences. Paris: Edition de l'Organisation.

Martin- Kniep, Giselle O. (2001). Portfolios del desempeño de maestros, profesores y directivos. La sabiduría de la Práctica. Buenos Aires Argentina:.Editorial Paidos.

Mora Vicente, Luis. Pangea. (2006). Internet, blogs y comunicación en un mundo nuevo. Un manual de supervivencia en la selva digital. Sevilla España: Fundación José Manuel Lara.

Prendes, Ma Paz. (2009). Referencia EA2009-0133. Competencias TIC para la docencia en la Universidad Pública Española: Indicadores y propuestas para la definición de buenas prácticas de la Universidad de Murcia.

Poisson, Daniel. (1998). Conférence de «Apprendre en autonomie avec les nouvelles technologies: nouveau rôle de l'enseignant». Les nouvelles technologies de communication et l'accès aux savoirs. Reims, 7 octobre.

Robbins. Stephen. (2004). Comportamiento organizacional. Teoría y práctica. México: Prentice-Hall Hispanoamericana, $10^{\mathrm{a}}$ ed.

Stieres M., Lea, (2000). The personal in university writing: uses of reflective learning journals, en B: Students Writing in Higher Education: Nex Contextes, Buckinghan. Open University Press. PAG. 61. Créme.

Watzlawick, Paul. (1978). La réalité de la réalité. Point Essais. Confusion, desinformation, communication. Francia: Seuil. 


\section{Webgrafía}

http://www.um.es/competenciastic/index.html

http://www.um.es/competenciastic/indicadores.html

http://www.um.es/competenciastic/autoevaluacion.html

http://peremarques.pangea.org/docentes.htm

http://www.scribd.com/doc/2553283/INFORME-BRUNDTLAND 